\title{
The missing climate forcing
}

\author{
J. HANSEN, M. SATO, A. LAGIS And R. RUEDY \\ NASA Goddard Institute for Space Studies, 2880 Broadway, New York, NY 10025, USA
}

\begin{abstract}
SUMMARY
Observed climate change is consistent with radiative forcings on several time scales for which the dominant forcings are known, ranging from the few years after a large volcanic eruption to glacial-tointerglacial changes. In the period with most detailed data, 1979 to the present, climate observations contain clear signatures of both natural and anthropogenic forcings. But in the full period since the industrial revolution began, global warming is only about half of that expected due to the principal forcing, increasing greenhouse gases. The direct radiative effect of anthropogenic aerosols contributes only little towards resolving this discrepancy. Unforced climate variability is an unlikely explanation. We argue on the basis of several lines of indirect evidence that aerosol effects on clouds have caused a large negative forcing, at least $-1 \mathrm{Wm}^{-2}$, which has substantially offset greenhouse warming. The tasks of observing this forcing and determining the microphysical mechanisms at its basis are exceptionally difficult, but they are essential for the prognosis of future climate change.
\end{abstract}

\section{INTRODUGTION}

A radiative forcing is a change imposed on the planetary radiation balance which tends to alter global temperature, for example, a change of incoming solar irradiance or a change of atmospheric composition. Such climate forcings are commonly measured in $\mathrm{Wm}^{-2}$ averaged over the earth. If the forcing is calculated after allowing for the rapid adjustment of stratospheric temperature, its value provides a good measure of the forcing's potential impact on global average temperature. That is, despite uncertainty in climate feedback processes and thus in climate sensitivity, for most radiative mechanisms two forcings of equal magnitude are expected to yield similar global temperature change (Hansen et al. 1997 a).

Understanding of past climate change and projection of future changes depend on knowledge of climate forcings and climate sensitivity. Indeed, these are the principal quantities which must be known, along with the magnitude of unforced climate variability, to assess the importance of human influences on climate. We argue that on certain timescales the dominant climate forcings are known to a reasonable accuracy, providing opportunity for empirical verification of climate sensitivity. But on the timescales crucial to assessment of human influence, decades to centuries, we conclude that at least one of the principal climate forcings is not being measured. Absence of these data prevents reliable projection of future climate change and assessment of the effectiveness of any strategies for mitigating human influence on climate.

\section{INDIVIDUAL VOLGANOES}

A large volcanic eruption, by injecting a massive amount of $\mathrm{SO}_{2}$ into the stratosphere which subsequently forms a global layer of sulphuric acid aerosols, can cause a climate forcing which clearly dominates other global radiative forcings for $1-2$ years following the eruption. Aerosols from the 1991 eruption of Mount Pinatubo, for example, reflected to space sufficient sunlight to cause a (negative) forcing of about $3 \mathrm{Wm}^{-2}$ (Minnis et al. 1993; Hansen et al. 1996 b). By comparison, none of the other natural and anthropogenic radiative forcings that we know about, such as changes of solar irradiance or greenhouse gases, alters the radiative forcing by more than $0.1 \mathrm{Wm}^{-2}$ on a twoyear timescale.

Thus it is appropriate to search climatic records for a climate response following large volcanic eruptions, but two factors tend to frustrate this task. First, the large thermal inertia of the ocean limits the response to a brief forcing to a small fraction of what would occur for a long-lived forcing. For example, climate models calculate a maximum cooling of about $0.5^{\circ} \mathrm{C}$ following Pinatubo, while the same models would yield a cooling of 3-4 ${ }^{\circ} \mathrm{C}$ if the aerosols remained present indefinitely. Second, unforced (chaotic) climate variability on the interannual timescale can be as much as a few tenths of a degree, thus hindering detection of a deterministic climate response. As a consequence, the best chances for quantitative empirical analysis are (1) examination of the mean climate response after all the large volcanoes in the period of global climatic data, and (2) specific analysis of Pinatubo, the volcano with the greatest aerosol optical depth in this century as well as the best climate observations. The composite technique has been used previously by Mitchell (1961), Mass \& Schneider (1978) and Self \& Rampino (1988).

Figure 1 shows the observed mean global temperature change near the times of the five volcanoes producing the largest optical depths since 1880, based on temperature data of Hansen et al. (1996a). We conclude that there is clear evidence for cooling following large volcanoes, but because of uncertainties in the measured temperatures and in the definition of zero temperature anomaly prior to the eruption, the 


\section{Composite Response to Five Largest Volcanoes since 1880}

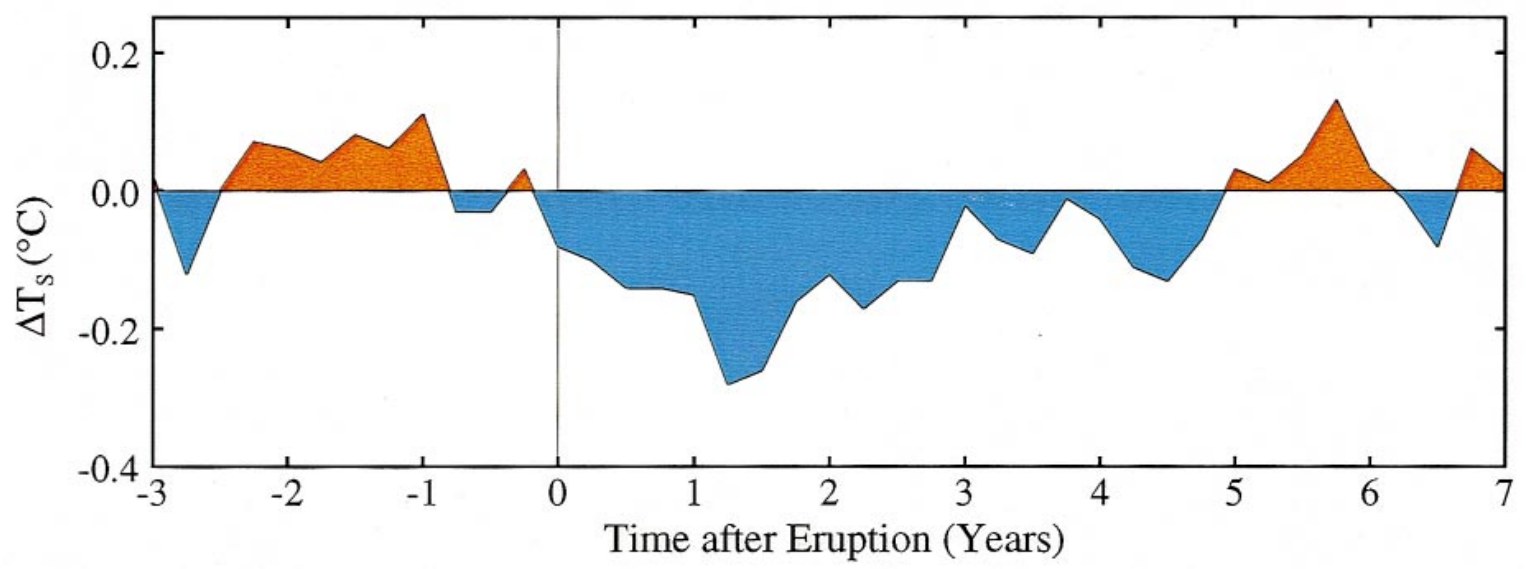

Figure 1. Global surface temperature change near the time of the five volcanoes producing the greatest optical depths since 1880: Krakatau (1883), Santa Maria (1902), Agung (1963), El Chichon (1982) and Pinatubo (1991). Temperature data, from Hansen et al. $(1996 a)$, are three-month means. Base period defining the zero temperature anomaly is the year preceding the eruption.

magnitude of the mean cooling is only confined to a range of about $0.25 \pm 0.1^{\circ} \mathrm{C}$ (Hansen et al. $1996 b$ ).

The most quantitative comparison of observations with climate models is provided by Pinatubo, for which a maximum cooling of $0.5^{\circ} \mathrm{C}$ was predicted for the summer and fall of the year following the eruption. Observations were found to be in good agreement with this prediction (Parker et al. 1996). Observed annual mean cooling of about $0.3^{\circ} \mathrm{C}$ was somewhat smaller than calculated, as shown in $\S 4$ below, but overall this natural climate experiment provided a clear indication of thermal response to the radiative forcing.

Volcanic aerosols thus provide useful empirical verification that the climate responds as expected to a radiative forcing. But they do not provide a good discriminate of climate sensitivity, because of the brevity of the forcing (Hansen et al. 1993). The reason for this is that feedback processes such as changes of water vapour, clouds, and sea ice (which largely determine long-term climate sensitivity) come into play only in proportion to temperature change, which is damped to a small fraction of its equilibrium response by the ocean's thermal inertia (Hansen et al. 1984). A good empirical measure of climate sensitivity requires a forcing that remains present for at least a century.

\section{PALAEOGLIMATE}

A complement to volcanic aerosols is provided by palaeoclimate radiative forcings. The causes of climate changes from ice ages to interglacial periods are uncertain. These climate fluctuations tend to be associated with periodic changes of the Earth's orbit (Hayes et al. 1976), but the mechanisms of change continue to be debated. However, for the purpose of inferring climate sensitivity, it is irrelevant what ultimately causes the climate change.

The important point is that climate sensitivity to a radiative forcing can be inferred by comparing the Earth's radiation balance during ice ages and

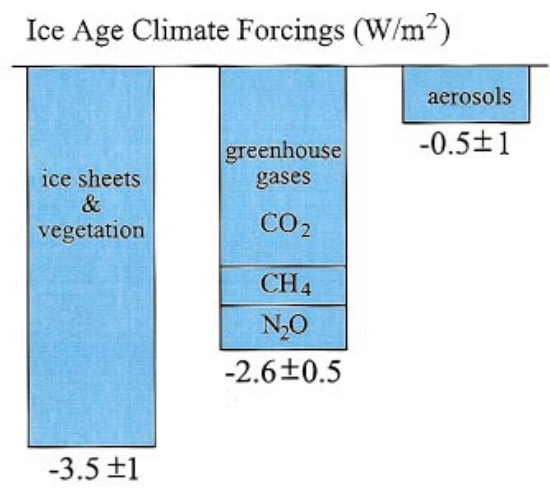

Figure 2. Global radiative forcings during the last ice age relative to the current interglacial period. The total forcing is $-6.6 \pm 1.5 \mathrm{Wm}^{-2}$. Thus the $5{ }^{\circ} \mathrm{C}$ cooling of the ice age implies a climate sensitivity of $0.75^{\circ} \mathrm{C}$ per $1 \mathrm{Wm}^{-2}$ forcing.

interglacial periods (Hansen et al. 1984, 1993; Hoffert \& Covey 1992). Averaged over several centuries the planet must be in radiation balance within a fraction of $1 \mathrm{Wm}^{-2}$, as can be verified by calculating the energy rate associated with disequilibrium processes such as melting of continental glaciers or changes in ocean temperature.

The changes in the Earth's atmosphere and surface which would alter the planet's radiation balance during the last major ice age are known with reasonable confidence, based on measurements of the composition of the ice age atmosphere (Lorius et al. 1990) and reconstructions of planetary surface conditions (CLIMAP 1981). The radiative forcings maintaining the ice age cold were: increased reflection of sunlight by the continents due to larger ice sheets and vegetation changes, decreased amounts of the greenhouse gases $\mathrm{CO}_{2}, \mathrm{CH}_{4}$ and $\mathrm{N}_{2} \mathrm{O}$, and increased atmospheric aerosol particles (Hansen et al. 1984, 1993; Hoffert \& Covey 1992). These surface and atmospheric changes caused a total forcing of $-6.6 \pm 1.5 \mathrm{Wm}^{-2}$ (figure 2).

Given knowledge of the radiative forcing, measurements of the temperature change between the glacial 
(a)
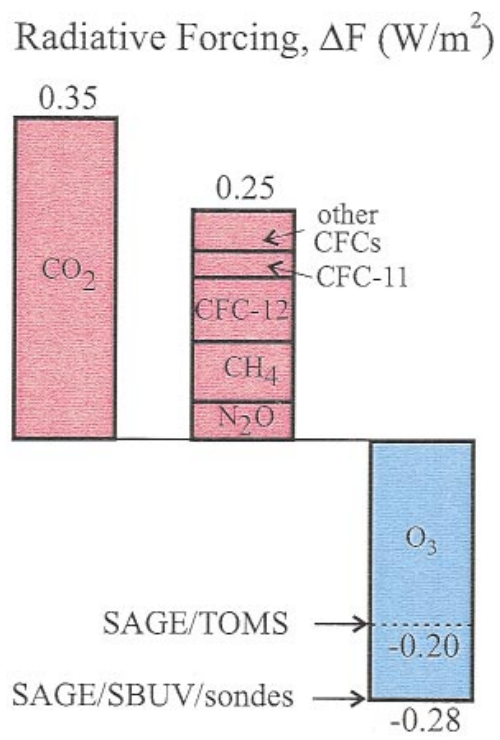

(b)
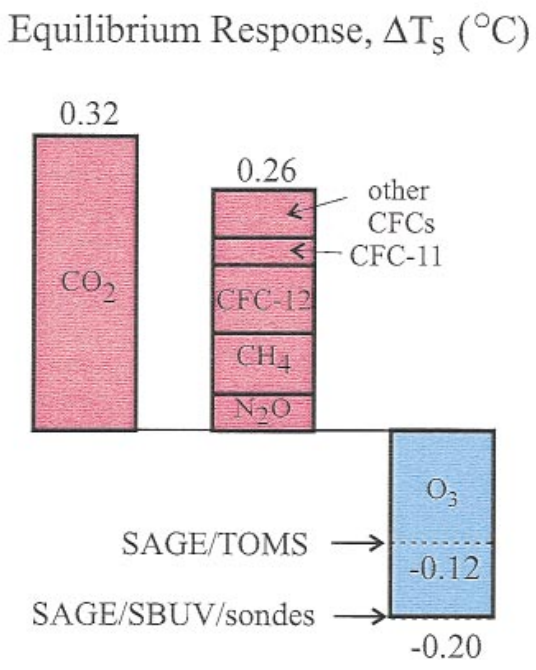

Figure 3. (a) Adjusted radiative forcings for greenhouse gas changes over the period 1979-1994, based on abundance changes specified by Hansen et al. (1997a). (b) Equilibrium surface air temperature changes for these same greenhouse gas changes, as calculated with a GCM having climate sensitivity $3.8{ }^{\circ} \mathrm{C}$ for doubled $\mathrm{CO}_{2}($ Hansen et al. 1997 a).

and interglacial climates provide an empirical measure of climate sensitivity. This empirical measure includes the 'fast' feedback processes (Hansen et al. 1984), such as changes in water vapour, clouds and sea ice cover, which respond quickly to changed temperature and are included in current global climate models. But the empirical data also include any other feedbacks that may exist in the real world, such as biogenic aerosols and aerosol effects on clouds (Charlson et al. 1987), as well as systematic alterations of the ocean's circulation.

The difficulty in obtaining an accurate empirical climate sensitivity from this palaeoclimate test, until recently, has been uncertainty in the tropical temperature during the last ice age. CLIMAP (1981) reconstructions had low latitude ocean temperatures little different than today's climate, while land data indicated a cooling of $3-5{ }^{\circ} \mathrm{C}$ in much of the tropics (Rind \& Peteet 1985). But recent data (Guilderson et al. 1994; Schrag et al. 1996) indicate that significant tropical cooling did occur during the ice age. Rather than the $3.7^{\circ} \mathrm{C}$ global cooling implied by CLIMAP SSTs (Hansen et al. 1984), a better estimate of the global mean ice age cooling is $5 \pm 1{ }^{\circ} \mathrm{C}$. Thus the climate sensitivity implied by the last ice age is about $5^{\circ} \mathrm{C} /\left(6.6 \mathrm{Wm}^{-2}\right)=0.75^{\circ} \mathrm{C}$ per $\mathrm{Wm}^{-2}$, equivalent to $3 \pm 1^{\circ} \mathrm{C}$ for the canonical doubled $\mathrm{CO}_{2}$ forcing $\left(4.2 \mathrm{Wm}^{-2}\right)$. This empirical sensitivity is in the middle of the broader range, $1.5^{\circ} \mathrm{C}$ to $5^{\circ} \mathrm{C}$ for doubled $\mathrm{CO}_{2}$, suggested by global climate models (Dickinson 1985).

The most uncertain ice age forcing is perhaps that of aerosols. Our present estimate, $-0.5 \mathrm{Wm}^{-2}$, is less than the $-1 \mathrm{Wm}^{-2}$ we used earlier (Hansen et al. 1993) because of evidence that desert dust causes a small warming (Overpeck et al. 1996 and §5 below). Although palaeoclimate changes of aerosols and temperature are recorded in ice cores, satisfactory analysis will require data from several locations because of the heterogeneous distributions of different aerosol types.
We conclude that palaeoclimate data confirm that the climate system responds in a major way to global radiative forcings; indeed, the data yield an invaluable empirical measure of climate sensitivity. Note that the distinction between climate forcings and feedbacks is flexible in climate analyses, and appropriately so, as it depends upon the timescale considered and the intended applications. But we have shown that when we specify as forcings those quantities that are normally taken as fixed boundary conditions in current global climate models, the empirical climate sensitivity is consistent with the sensitivity calculated by the models.

\section{REGENT YEARS : 1979-1995}

The period with the most precise climate forcing data began with the launch of the Nimbus 7 satellite, near the end of 1978. This satellite included instruments measuring solar irradiance, stratospheric aerosols, and ozone, measurements which subsequently have been continued on other satellites. Changes of the well-mixed greenhouse gases, such as $\mathrm{CO}_{2}, \mathrm{CH}_{4}, \mathrm{~N}_{2} \mathrm{O}$ and the chlorofluorocarbons (CFGs), are known accurately from measurements at a few ground stations. Other potentially significant forcings remain practically unmeasured, especially tropospheric aerosols and related cloud changes, and the ozone data is not adequate to define its forcing precisely. Nevertheless, this is the period with by far the most accurately defined radiative forcings, and it is possible that they represent most of the net forcing in that period. Thus, it is worthwhile to make a quantitative comparison of the measured forcings and the observed climate change during this period.

We first calculate the radiative forcings for the greenhouse gas changes used by Hansen et al. (1997a) for the period 1979-1994, e.g. $\Delta \mathrm{CO}_{2}=21.5 \mathrm{ppm}$, $\Delta \mathrm{CH}_{4}=162 \mathrm{ppb}$, and $\Delta \mathrm{N}_{2} \mathrm{O}=10.5 \mathrm{ppb}$. The well- 
mixed greenhouse gases yield a forcing of $0.6 \mathrm{Wm}^{-2}$ for this period (figure $3 a$ ). Ozone change is calculated to cause a substantial negative forcing in this period $\left(-0.2\right.$ to $\left.-0.3 \mathrm{Wm}^{-2}\right)$, but its value is uncertain because it depends sensitively on the poorly defined ozone change in the tropopause region.

We use two alternative ozone change profiles, defined by Hansen et al. (1997a). Both profiles employ the $\mathrm{O}_{3}$ depletion in the tropopause region measured by the SAGE satellite instrument (McCormick et al. 1992, updated by J. Zawodny, personal communication). This depletion occurs at the lower limit of the SAGE sensitivity, and the reality of SAGE data at low latitudes has been questioned on the grounds that large depletion is not expected there. But there is evidence that cirrus clouds, which are abundant at the altitudes and latitudes in question, may activate ozone depletion (Borrmann et al. 1996; Reichardt et al. 1996). At any rate, as a sensitivity study, for the want of better measurements, we take the SAGE data at face value.

Figure $3 b$ gives the equilibrium temperature changes calculated to result from the radiative forcings, based on a GCM having equilibrium sensitivity $3.8^{\circ} \mathrm{C}$ for doubled $\mathrm{CO}_{2}$ (Hansen et al. 1997 a). The responses have similar proportionality to forcings for the wellmixed greenhouse gases, but the response to the ozone radiative forcing is relatively less.

The net equilibrium temperature change calculated for all greenhouse gas changes in the 15 -year period is $0.4^{\circ} \mathrm{C}$. Only a fraction of this warming could be realized in the 15-year period because of the finite response time of the climate system. Indeed, with climate sensitivity $3.8^{\circ} \mathrm{C}$ for doubled $\mathrm{CO}_{2}$, it requires several decades to reach $50 \%$ of the equilibrium response (Hansen et al. 1984). To analyse the transient response it is necessary to include the thermal inertia effects of the mixed layer and the deeper ocean.

Simulations of the transient climate response have been carried out for the period 1979-1995 by Hansen et al. $(1997 \mathrm{~b})$, employing greenhouse gas forcing and measured changes of stratospheric aerosols and solar irradiance. The individual forcings employed are shown in figure $4 a$ as a function of time. The forcings are added one-by-one, cumulatively, to the GISS atmospheric GCM attached to a Q-flux ocean model. An ensemble of five runs is made for each set of forcings. Each curve shown here is the average of five runs.

The simulated global mean surface temperature is compared with observations in figure $4 b$. The modelled and observed temperatures have a high correlation, indeed the correlation of the five-run ensemble mean based on all four radiative forcings with the observations is almost as high as the mean of the correlations between the individual runs and the mean of the other runs (Hansen et al. 1997 b). Of course the model cannot reproduce features associated with ocean dynamics, namely El Niño warming in 1983 and La Niña cooling in 1989. Because of the brevity of this period, the observed surface temperature change cannot provide convincing evidence of climate response to radiative forcings, except perhaps for the cooling following the 1991 Pinatubo eruption.
Results for the troposphere and stratosphere are shown in figure $4 c$ and $4 d$, where the observations are based on MSU (Microwave Sounding Unit) channels 2 and 4, respectively (Spencer \& Christy 1993). The tropospheric result is similar to that at the surface, but, at least in the model, the ozone change reduces the warming trend, and, in the observations, the fluctuations associated with El Niños and La Niñas are enhanced. The results in the stratosphere, with warming after both El Chichon and Pinatubo and a long-term cooling trend (the latter caused, at least in the model, mainly by ozone depletion), present evidence of climate response to both natural and anthropogenic radiative forcings. The correspondence of observed stratospheric change with ozone forcing is shown in more detail by Ramaswamy et al. (1996).

The significance of temperature trends for such a short period is limited for two reasons. First, unforced variability, or chaos, can significantly influence a short trend, as quantified in figure 1 of Hansen et al. (1995c). This chaotic factor can be diminished in the climate model via an ensemble of model runs, but the Earth only runs through the 'experiment' period once, so unforced variability is an inherent limitation of a short period. Second, the brevity of the period increases the relative importance of any 'unrealized response' to climate forcings which were introduced in previous decades but which are still having an effect on climate change due to the lag associated with the ocean's thermal inertia. The lag effect is negligible for the stratosphere, which has a rapid radiative relaxation time and only weak coupling with the troposphere, but it is important for the troposphere and surface. We obtain an approximation of the lag effect by including a constant $0.65 \mathrm{Wm}^{-2}$ additional forcing, which is an estimate of the initial disequilibrium forcing (Hansen et al. 1997 b).

Proper analysis of the surface and tropospheric temperature trends requires simulations that begin much earlier, preferably prior to the period of significant forcings, but such simulations require additional assumptions, especially regarding climate forcings. We (Hansen et al. 1993) and others (Mitchell et al. 1995) have carried out transient GGM simulations covering the period from the 1800 s to the present. It is possible to obtain surface temperature trends reasonably consistent with observations over both the past century and the past few decades; but the results are dependent on rather arbitrary assumptions about aerosol or cloud changes, which are needed to provide a negative forcing to reduce the warming caused by greenhouse gases. In the following section we provide a simplified summary analysis of the required century timescale forcing.

We conclude that data for recent years, 1979-1995, exhibit evidence of climate responses to both natural and anthropogenic climate forcings. The brevity of the period limits interpretation, especially because of the influence of unforced climate variability on trends. This difficulty will decrease rapidly as the period of detailed data lengthens (Hansen et al. 1995c). But ambiguity in interpretation will persist as long as major climate forcings are unmeasured. 


\section{INDUSTRIAL PERIOD: THE MISSING FORGING}

Let us estimate the change of radiative forcings over the industrial era, and the equilibrium surface temperature changes that would be expected to accompany those forcings. In estimating the temperature change, we account for the fact that all forcings are not equivalent.

Tropospheric aerosols deserve special attention because they are one of the more uncertain forcings and because they illustrate the sometimes complex relation between forcing and response. Figure $5 a$ shows the radiative forcing for several aerosols as a function of their single scatter albedo, $\varpi$. The forcing was calculated with the sector version of the GISS GGM (Hansen et al. 1997a) because of its computational efficiency, but test calculations with the current complete GISS GCM gave practically identical results. The 'sulphate' aerosols use the size distribution of Lacis et al. (1992), which has effective radius $\approx 0.5 \mu \mathrm{m}$. The value of $\varpi$ indicated for sulphates is used only at those wavelengths where it is less than $\varpi$ for pure sulphuric acid, i.e. essentially at all wavelengths less than $3 \mu \mathrm{m}$.

Our industrial 'sulphates' are intended to represent the entire anthropogenic industrial haze. Thus the appropriate $\varpi$ must account for all absorbing constituents, whether they occur as impurities in sulphate particles or as separate particles. In situ measurements and calculations (references given by Haywood \& Shine (1995) and Hansen et al. (1997a)) typically give $\varpi \approx 0.88-0.96$, even in regions far from the aerosol sources. R. Charlson (personal communication) argues that the observations exaggerate absorption because the measurement techniques evaporate the conservatively scattering liquid particles. Recent model calculations (plate 3 of Liousse et al. 1996 ) yield $\varpi$ similar to the values we employ. More extensive observations of the dependence of $\varpi$ on all anthropogenic sources are needed.

The surface air temperature change as a function of $\varpi$, as calculated by the GISS sector GCM, is shown in figure $5 b$. The important point is that the effectiveness of tropospheric aerosols as a cooling mechanism is reduced by aerosol absorption more than would be estimated from the radiative forcing. The reason is the 'semi-direct' aerosol effect on clouds, by which aerosol warming slightly reduces large scale cloud cover in the atmospheric layer with absorbing aerosols (Hansen et al. $1997 a$ ). The magnitude of the semi-direct aerosol effect is model-dependent, as it depends on the representation of cloud physics: thus, it needs to be studied with other GCMs. But the physical basis by which aerosol heating reduces relative humidity and large scale cloud cover is straightforward, so we believe that the sense we find for this effect is correct.

Biomass combustion aerosols cool global surface air temperature $\left(T_{\mathrm{s}}\right)$ more effectively than industrial sulphates of the same $\varpi$, because the biomass aerosols occur more at low latitudes over low albedo ocean. The sulphate and biomass aerosol distributions which we employ are specified in figure 16 of Hansen et al.
(1993). Desert aerosols, based on the distribution, sizes and $\varpi$ (global mean $\varpi \approx 0.9$ ) of Tegen et al. (1996), yield a forcing of $0.3 \mathrm{Wm}^{-2}$. But the equilibrium global temperature change for these desert aerosols is only $+0.2^{\circ} \mathrm{C}$. The difference in this response, compared to sulphate aerosols, is due to the vertical distribution of desert aerosols of Tegen et al., which was generated by a three-dimensional transport model and has a large portion of the aerosols in the middle and upper troposphere. Following Tegen et al., we assume that half of the dust aerosols are anthropogenic, and thus we reduce the calculated forcing and response by one half.

Let us now use the same approach to calculate the radiative forcings and equilibrium temperature changes for all the climate forcing mechanisms of which we are aware (figure 6). The well-mixed greenhouse gases yield $2.5 \mathrm{Wm}^{-2}$ and $2.4^{\circ} \mathrm{C}$. We use the IPCG (1996) estimates for preindustrial greenhouse gases, and our calculated forcings agree with IPCC (1996). The tropospheric $\mathrm{O}_{3}$ change from the preindustrial era to 1980, based on Crutzen (1994), yields $+0.3 \mathrm{Wm}^{-2}$ and $+0.3{ }^{\circ} \mathrm{C}$. The ozone change of the past 15 years, based on the $\mathrm{SAGE} / \mathrm{SBUV} /$ sondes data discussed above, yields $-0.3 \mathrm{Wm}^{-2}$ and $-0.2{ }^{\circ} \mathrm{C}$. The estimated solar irradiance change, a $0.16 \%$ increase in 200 years based on the reconstruction of Lean et al. (1995), yields $+0.3 \mathrm{Wm}^{-2}$ and $+0.2^{\circ} \mathrm{C}$.

We consider tropospheric aerosol changes in three categories. For industrial aerosols, which are mainly fossil fuel sulphates and include soot and other impurities that reduce $\varpi$, we take $\varpi=0.95$ and (global mean) $\tau=0.017$ (Charlson et al. 1991; this estimate for $\tau$ refers to anthropogenic sulphate, but $\tau$ would not be greatly altered by the absorbing impurities). For biomass combustion aerosols we take, as suggested by Penner et al. (1992), $\varpi=0.92$ and global mean $\tau=$ 0.017; however, we reduce the calculated forcing and response by one-third to account for the existence of pre-industrial biomass combustion. For desert aerosols we use the properties of Tegen et al. (1996). The desert aerosol forcing and response has considerable uncertainty, because of poorly defined optical properties (Sokolik \& Toon 1996) and uncertainty in the aerosol vertical distribution. The sum of all these anthropogenic aerosols is a forcing of $-0.3 \mathrm{Wm}^{-2}$ and an equilibrium response of $-0.1^{\circ} \mathrm{C}$.

What other potential radiative forcings can be envisioned? Considering the Earth as a planet seen from space, it is apparent that clouds are the dominant factor influencing both solar heating of the planet and thermal emission to space. Thus even slight anthropogenic cloud changes may represent a significant forcing. As such cloud changes are unmeasured, we can only examine them quantitatively in indirect ways. But from the perspective of space there are also obvious anthropogenic alterations of the planetary surface, whose effect on the planetary radiation balance must be considered. The radiative forcing due to surface changes can be examined quantitatively, so we consider it first.

The data set of Matthews (1983) for current global land use patterns has been inserted into the GISS GCM, as a replacement of the 'natural' vegetation, by 


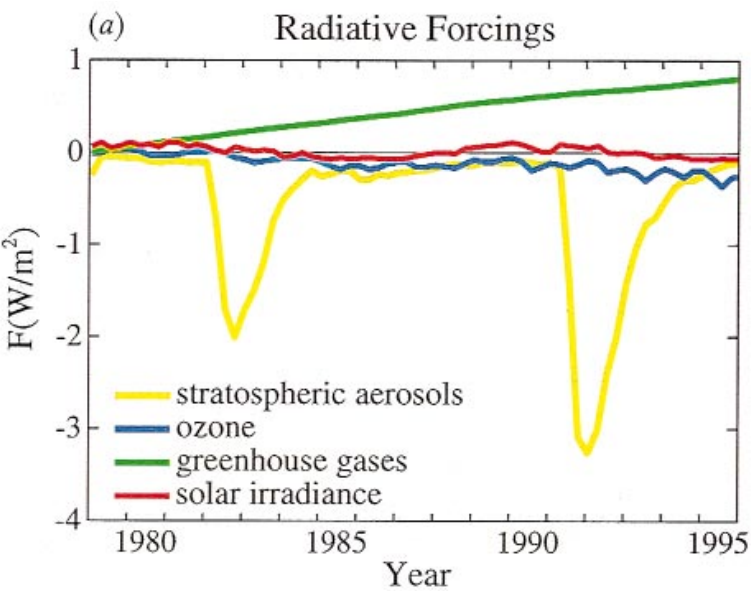

(b)

Surface Temperature

(c) Tropospheric Temperature
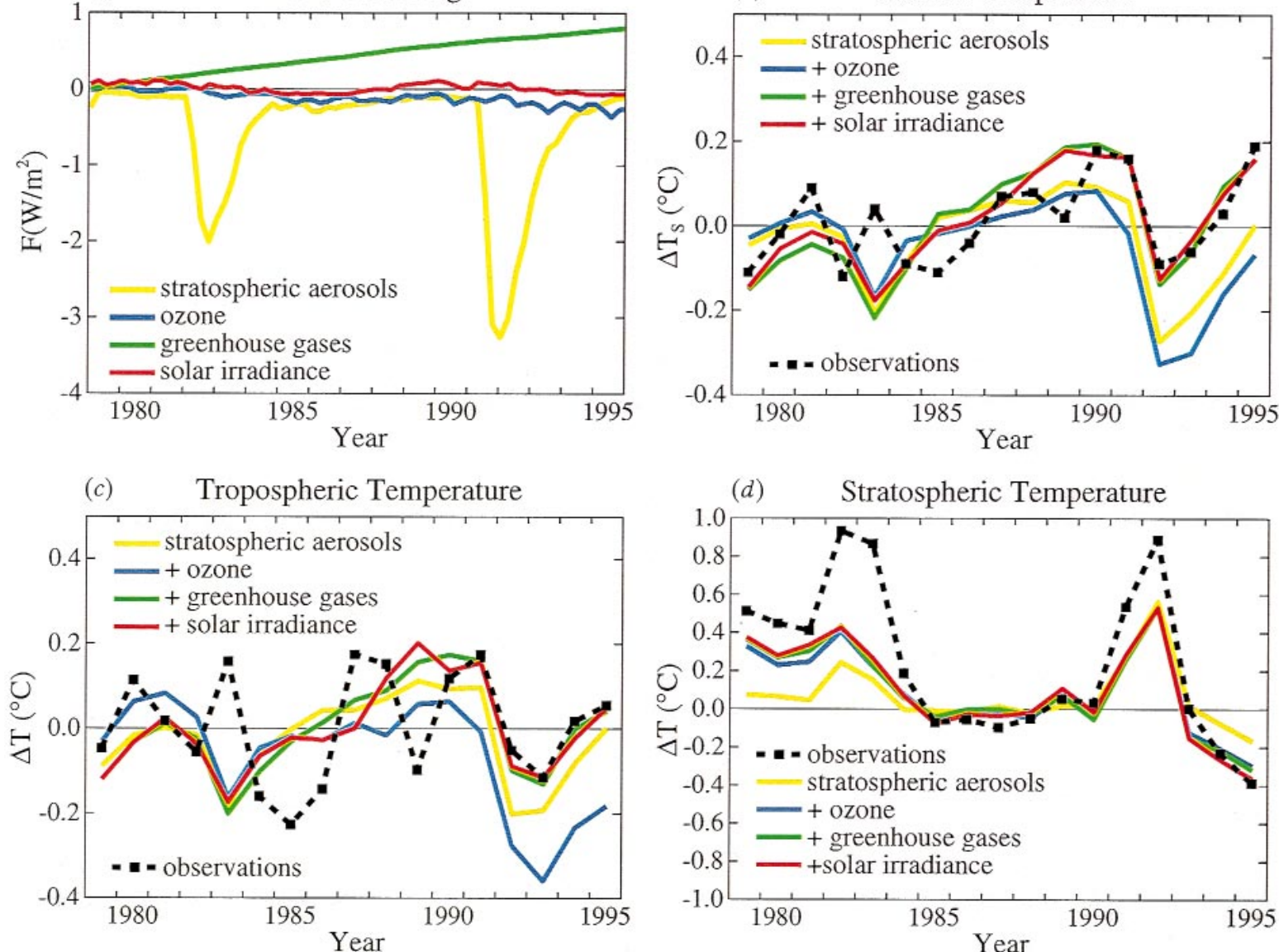

(d) Stratospheric Temperature

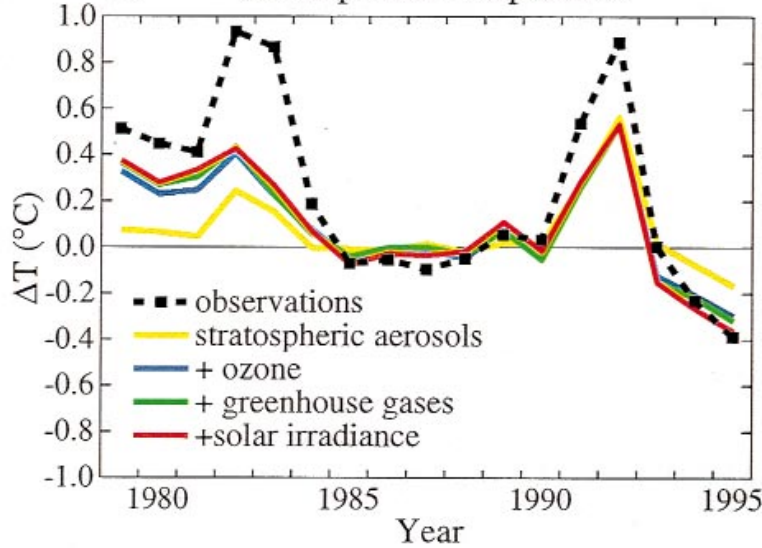

Figure 4. (a) Radiative forcings since 1979 due to changes of stratospheric aerosols, ozone, well-mixed greenhouse gases and solar irradiance. $(b, c, d)$ Observed global annual mean surface, tropospheric and stratospheric temperature changes, and GCM simulations as the successive radiative forcings are added one-by-one, cumulatively. An additional constant $+0.65 \mathrm{~W} / \mathrm{m}^{2}$ forcing is included in all GCM simulations, representing the estimated disequilibrium forcing for 1979. Base period defining zero mean observed temperature is 1979-1990 for the surface and the troposphere, and 1984-1990 for the stratosphere.
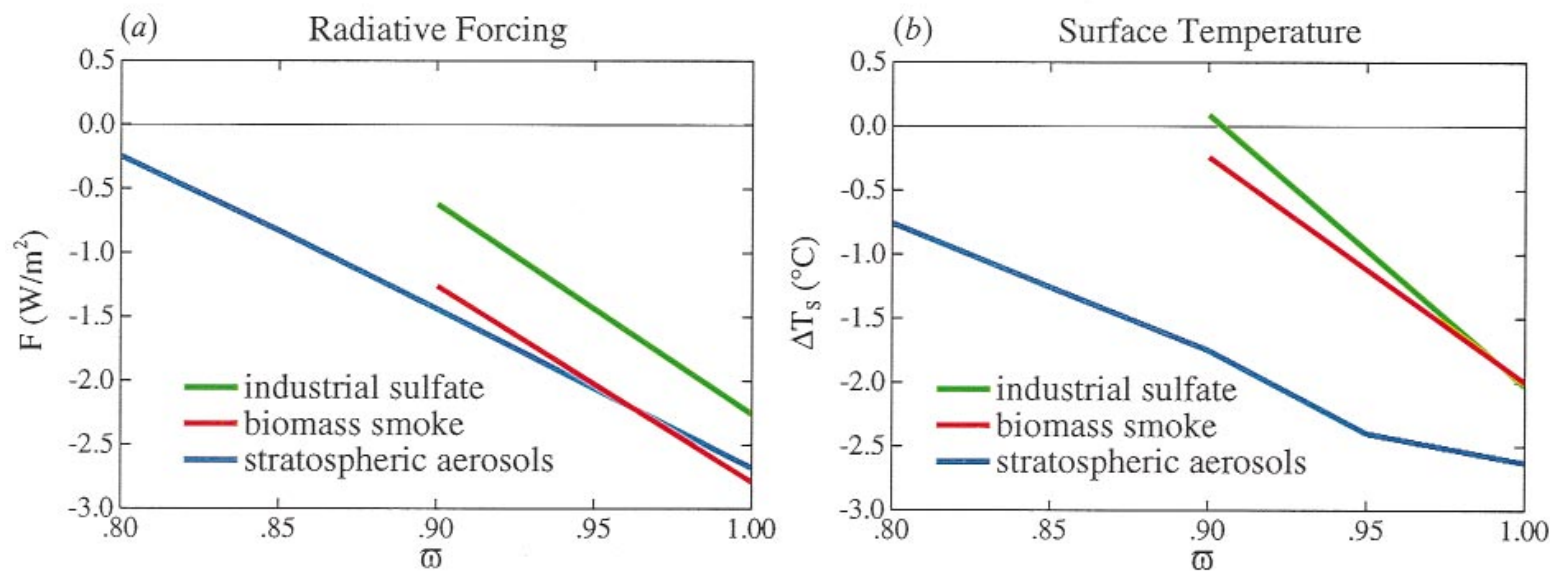

Figure 5. Radiative forcings $(a)$ and equilibrium global surface air temperature sensitivity $(b)$ for different aerosols $(\Delta \tau=0.1)$ as a function of their single scatter albedo, as calculated with a sector (Wonderland) GCM (Hansen $e t$ al. $1997 a)$; the GCM has sensitivity $3.8^{\circ} \mathrm{C}$ for doubled $\mathrm{CO}_{2}$. Stratospheric sulphate aerosols have a uniform global distribution, while other aerosols are given distributions that mimic latitudinal and land/ocean variations estimated for the real world.

Hansen et al. (1997 b) to obtain a crude measure of the forcing due to surface changes. They find that current land use results in a global radiative forcing of $-0.4 \mathrm{Wm}^{-2}$ relative to prehuman natural vegetation.
The sense of the anthropogenic influence, cooling, is a result of a reduction in the area of forests, which are dark; the largest effect occurs during seasons with snow on the ground, as the greater 'masking depth' of trees 
(a)

Radiative Forcing, $\Delta \mathrm{F}\left(\mathrm{W} / \mathrm{m}^{2}\right)$

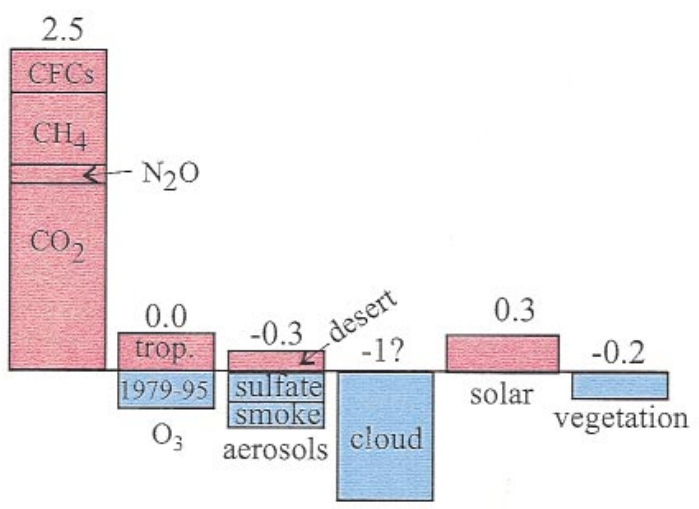

(b)

$$
\text { Equilibrium Response, } \Delta \mathrm{T}_{\mathrm{S}}\left({ }^{\circ} \mathrm{C}\right)
$$

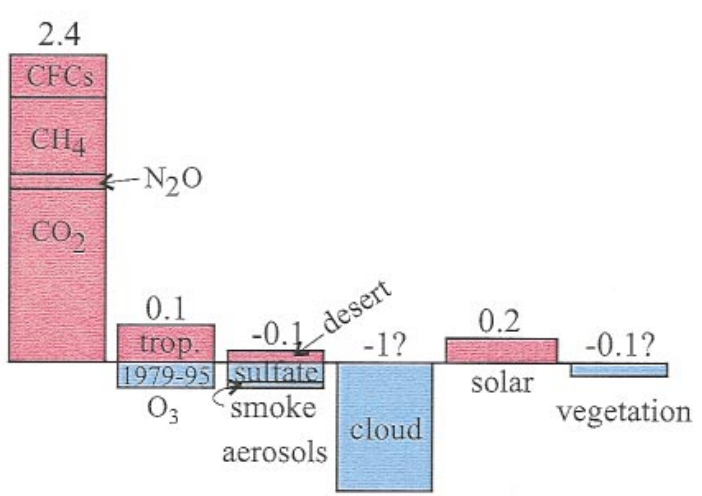

Figure 6. Radiative forcings $(a)$ and equilibrium surface air temperature changes $(b)$ calculated for atmospheric changes occurring between the pre-industrial era and 1995, the temperature changes being based on a GCM with sensitivity $3.8{ }^{\circ} \mathrm{C}$ for doubled $\mathrm{CO}_{2}$. Greenhouse gas changes are $\mathrm{CO}_{2}(280$ to $359 \mathrm{ppm}), \mathrm{CH}_{4}(700$ to $1730 \mathrm{ppb}), \mathrm{N}_{2} \mathrm{O}$

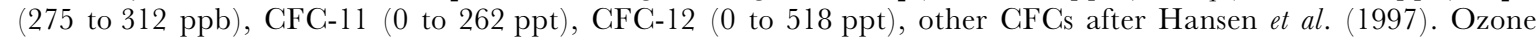
pre-industrial to 1980 change based on Crutzen (1994).
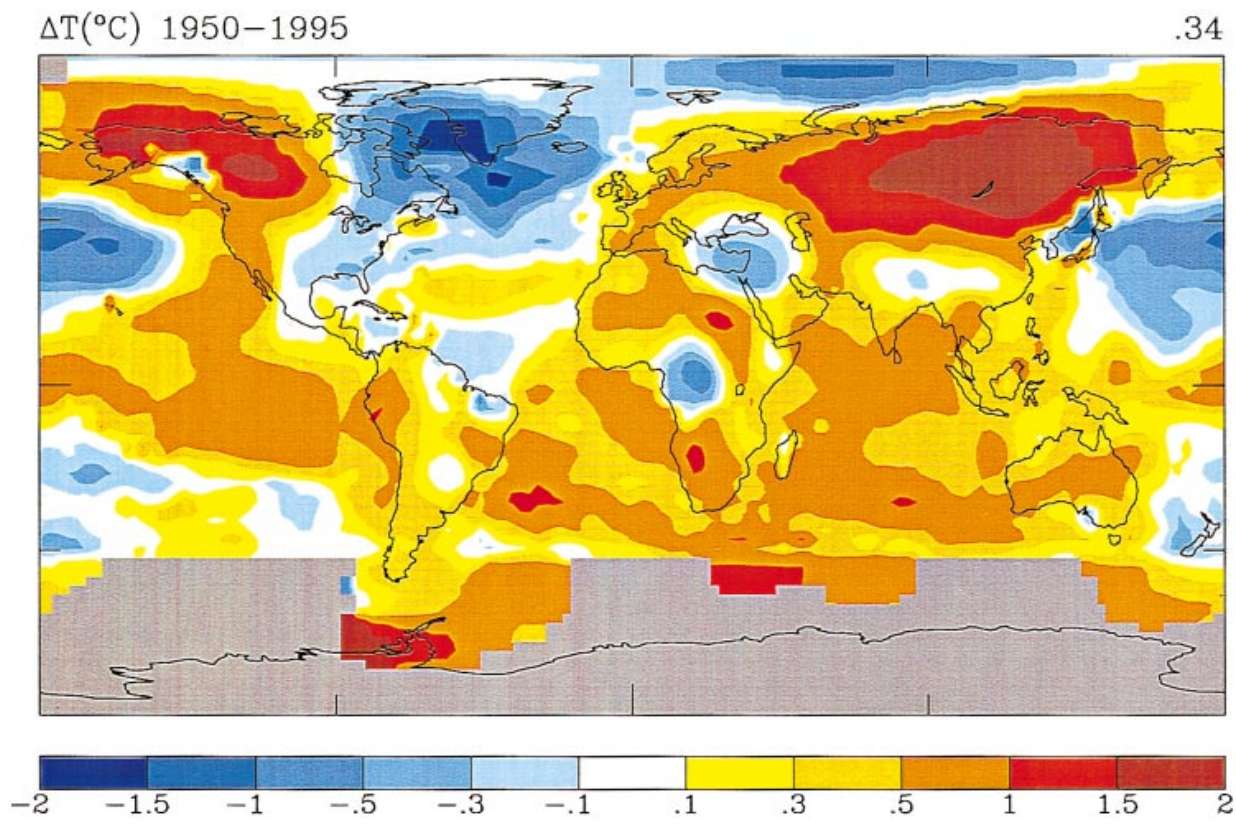

Figure 7. 1950-1995 change of surface temperature $\left({ }^{\circ} \mathrm{C}\right)$ based on linear trend. Data, from Hansen et al. (1996a), uses SST over the ocean and surface air temperature over land areas.

is more effective than crops at shielding surface snow from incident sunlight. This negative forcing occurs mainly in the United States, Western Europe, the former Soviet Union and the India-China region. We estimate that about half of this deforestation occurred before the industrial revolution. Thus in figure 6 we include a forcing of $-0.2 \mathrm{Wm}^{-2}$ for land use. Hansen et al. $(1997 a)$ find that surface albedo changes at latitudes less than $55^{\circ}$ are only half as effective as greenhouse gases in generating surface temperature change; thus we take $\Delta T_{\mathrm{s}} \approx-0.1{ }^{\circ} \mathrm{C}$. However, this should be computed with the GGM using the land use patterns of Matthews (1983).

Now let us consider the possible radiative forcing due to anthropogenic cloud changes. Because of the absence of cloud change observations adequate for a direct computation of the forcing, we consider three indirect pieces of evidence.

\section{(a) Evidence A: diurnal cycle of $T_{\mathrm{s}}$}

The first, and we believe the strongest, evidence is inferred from the observed damping of the diurnal cycle of surface air temperature that has occurred since 1950 in much of the world's land area (Karl et al. 1993). Many mechanisms dampen the diurnal cycle, including increase in soil moisture and increase in atmospheric aerosols. However, quantitative analysis (Hansen et al. 1995b) shows that these mechanisms are incapable of accounting for the large global-scale damping reported by Karl et al. (1993). Hansen et al. $(1995 b)$ find that, of all the mechanisms that have been 
proposed to account for the diurnal damping, the only one capable of producing such a large effect is an increase in cloud cover, with the increase occurring preferentially over land areas (or, stated differently, preferentially in the Northern Hemisphere). The mechanisms by which increased clouds damp the diurnal cycle is by decreasing solar heating of the surface in the day and decreasing surface cooling to space at night. Increasing aerosols contribute (a smaller amount) to the diurnal damping via the same mechanisms.

The observed diurnal cycle change does not imply the level at which the cloud cover change occurred. However, if we make the plausible assumption that the cloud change is an indirect effect of anthropogenic aerosols, that would suggest a change of low level clouds. Support for this inference is provided by the fact that the change of high clouds (which might be modified by aircraft) required to damp the diurnal cycle as observed is implausibly large (Hansen et al. $1995 b$ ). The increase in low cloud cover required to account for the observed diurnal damping is only about $1 \%$ of the area of the globe.

The simulations of Hansen et al. (1995b) yielding best agreement with the observed changes of the diurnal cycle had a negative forcing, due to the combination of increased clouds and aerosols, slightly more than $1 \mathrm{Wm}^{-2}$. Based on our present conclusion that the direct aerosol forcing is no more than a few tenths of $1 \mathrm{Wm}^{-2}$, it follows that the cloud forcing required for agreement with the observed damping of the diurnal cycle is about $-1 \mathrm{Wm}^{-2}$.

\section{(b) Evidence B: magnitude of global warming}

The second piece of evidence for a negative cloud forcing is the difference between observed global temperature change of the past century, estimated to be about $0.6^{\circ} \mathrm{C}$ (Hansen et al. $1996 a$; IPCG 1996), and the temperature change expected due to known radiative forcings. The latter can be inferred from figure 6, which, excluding cloud changes, yields a net forcing of $2.3 \mathrm{Wm}^{-2}$. The resulting equilibrium global warming is $2.5^{\circ} \mathrm{C}$, if climate sensitivity is $3.8^{\circ} \mathrm{C}$ for doubled $\mathrm{CO}_{2}$. Alternatively, if climate sensitivity is $2{ }^{\circ} \mathrm{C}$ for doubled $\mathrm{CO}_{2}$, the lowest sensitivity consistent with palaeoclimate data, the equilibrium warming is $1.3^{\circ} \mathrm{C}$.

These equilibrium warmings must be reduced to the fraction of the equilibrium response expected to occur by the 1990s for a realistic transient scenario of the forcings. That fraction, which is a function of climate sensitivity, can be estimated from the transient simulations of Hansen et al. (1993): these yield about $60 \%$ and $80 \%$ for climate sensitivities $3.8^{\circ} \mathrm{C}$ and $2{ }^{\circ} \mathrm{C}$, respectively. Thus the known forcings should have yielded a global warming between $2.5 \times 0.6$ and $1.3 \times 0.8$, i.e. between $1.5^{\circ} \mathrm{C}$ and $1{ }^{\circ} \mathrm{C}$. As the existence of the positive greenhouse gas forcing is beyond question, the simplest explanation for why the calculated warming exceeds the observed warming of $0.6^{\circ} \mathrm{C}$ is the presence of another forcing of at least $-1 \mathrm{Wm}^{-2}$.
This interpretation assumes that long-term global temperature changes are primarily a response to global radiative forcings. Alternatively, observed temperature changes may be largely unforced fluctuations. Although we cannot disprove that possibility, the variabilities found in coupled atmosphere-ocean models (Manabe \& Stouffer 1996) suggest that it is an improbable explanation.

\section{(c) Evidence C: regional cooling patterns}

The final evidence of negative radiative forcing that we consider is the pattern of regional temperature change in the past several decades. As pointed out by Karl et al. (1995), there is a correlation between regions of cooling and source regions of anthropogenic aerosols. This spatial correlation causes transient climate simulations using greenhouse gases plus sulphate aerosols to yield a positive 'fingerprint' (Santer et al. 1996), compared to observations, which is not found with simulations using greenhouse gases alone.

Figure 7 shows observed temperature change based on regional linear trends for the period 1950-1995. The strongest features, at high latitudes in the Northern Hemisphere, including intense cooling centered in Baffin Bay, are discussed by Hansen et al. (1996a) in relation to possible changes in cold-season atmospheric dynamics and an increasing greenhouse effect. The principal features of relevance here are the mid-latitude regions of relative cooling over the eastern United States, south-west Europe, and the Far East, which are all regions of strong anthropogenic sulphate production.

In our calculations (Hansen et al. 1993, 1997a), which employ a sulphate $\varpi=0.95$, aerosol cooling alone is not sufficient to overcome the greenhouse warming and produce the observed magnitude of regional cooling. Our preliminary interpretation is that this represents evidence of the need for an additional regional cooling mechanism, which could be provided by increased cloud cover and increased cloud brightness associated with aerosols.

Caveats that must accompany this conclusion include the imprecise knowledge of aerosol optical depth and $\varpi$. Also our calculations were made with idealized Wonderland geography. Other GAMs, e.g. Mitchell et al. (1995) use real world geography but mimic aerosol albedo by changing the ground albedo, and exclude crucial aerosol absorption. Clearly more realistic analyses are needed before the significance of regional cooling patterns can be ascertained.

\section{DISGUSSION: THE NEED FOR MEASUREMENTS}

We have presented evidence for the existence of a substantial unmeasured negative climate forcing. Such an inference has been made by many others in recent years, perhaps most prominently by Charlson et al. (1992). Our conclusion differs quantitatively and qualitatively from theirs, as we find that anthropogenic aerosols per se provide very little forcing. 
Our conclusion that the direct aerosol effect is small depends in part on our use of a single scatter albedo $\varpi$ less than unity for anthropogenic aerosols. But the values we have chosen, $\varpi=0.95$ for industrial haze and $\varpi=0.92$ for biomass smoke, do not appear to greatly exaggerate absorption. Both in situ observations and calculations suggest that the mean $m$ for anthropogenic aerosols cannot be much higher than that, and, indeed, may be less (cf. references given by Haywood \& Shine 1995; Ogren 1995; Hansen et al. 1997a; Hobbs et al. 1997). Our figure 5 can be used to determine the effect of alternate choices for $\varpi$, but such changes will not qualitatively alter our conclusions about climate forcings summarized in figure 6 .

Inference of a large but unmeasured climate forcing is an extremely unsatisfactory state of affairs, calling into question our ability to make any reliable projection of future global climate change. Without an understanding of the nature of this forcing and how it relates to anthropogenic emissions, it is impossible to assess the impact of alternative emissions policies.

A coordinated research program is required, including in situ field studies and aerosol and cloud modelling, but the fundamental need is measurement of the radiative forcings. Changes in aerosol and cloud properties must be measured globally, defining flux changes to a decadal precision of $1 / 4 \mathrm{Wm}^{-2}$, thus allowing useful comparison with greenhouse gas and other forcings.

Current satellite measurements do not approach this precision, but $1 / 4 \mathrm{Wm}^{-2}$ precision could be obtained for both aerosol and cloud properties from the combination of appropriate shortwave photopolarimetric and infrared interferometric measurements (Hansen et al. 1995 a; Mishchenko \& Travis 1997). Simultaneous mapping of aerosol and cloud properties, including short-term and interannual variations, should help reveal indirect aerosol effects on clouds. In addition, coordinated field measurements of aerosol properties will be needed, and detailed vertical layering of aerosols and clouds obtainable with lidar measurements would also be invaluable.

We thank Robert Charlson and Tad Anderson for comments on our draft manuscript and Jose Mendoza for drafting assistance. This work was supported by the NASA Climate, EOS and Aircraft Assessment programs.

\section{REFERENGES}

Borrmann, S., Solomon, S., Dye, J. E. \& Luo, B. 1996 The potential of cirrus clouds for heterogeneous chlorine activation. Geophys. Res. Lett. 23, 2133-2136.

Charlson, R. J., Lovelock, J. E., Andreae, M. O. \& Warren, S. G. 1987 Oceanic phytoplankton, atmospheric sulfur, cloud albedo and climate. Nature, Lond. 326, 665-661.

Charlson, R. J., Langner, J., Rodhe, H., Leovy, C. B. \& Warren, S. G. 1991 Perturbation of the Northern Hemisphere radiative balance by backscattering from anthropogenic sulphate aerosols, Tellus 43AB, 152-163.

Charlson, R. J., Schwartz, S. E ., Hales, J. M., Cess, R. D., Coakley, J. A., Hansen, J. E. \& Hofmann, D. J. 1992 Climate forcing by atmospheric aerosols, Science, Wash. 255, 423-430.
CLIMAP project members 1981 Seasonal reconstruction of the Earth's surface at the last glacial maximum. Geolog. Soc. Amer. map and chart series $\mathbf{M C}-\mathbf{3 6}$.

Crutzen, P. J. 1994 Global tropospheric chemistry. In Low temperature chemistry of the atmosphere (ed. G. K. Moortgat et al.), pp. 465-498. Berlin: Springer.

Dickinson, R. E. 1985 Climate sensitivity. Adv. Geophys. 28A, 99-129.

Guilderson, T. P., Fairbanks, R. G. \&. Rubenstone, J. L. 1994 Tropical temperature variations since 20000 years ago: modulating interhemispheric climate change. Science, Wash. 263, 663-665.

Hansen, J., Lacis, A., Rind, D., Russell, G., Stone, P., Fung, I., Ruedy, R. \& Lerner, J. 1984 Climate sensitivity: analysis of feedback mechanisms. Geophys. Mono. 29, 130-163.

Hansen, J., Lacis, A., Ruedy, R., Sato, M. \& Wilson, H. 1993 How sensitive is the world's climate? Nat. Geogr. Res. Explor. 9, 142-158.

Hansen, J., Rossow, W., Carlson, B., Lacis, A., Travis, L., Del Genio, A., Fung, I., Cairns, B., Mishchenko, M. \& Sato, M. 1995 a Low-cost long-term monitoring of global climate forcings and feedbacks. Clim. Change 31, 247-271.

Hansen, J., Sato, M. \& Ruedy, R. 1995 b Long-term changes of the diurnal temperature cycle: implications about mechanisms of global climate change. Atmos. Res. 37, 175-209.

Hansen, J., Wilson, H., Sato, M., Ruedy, R., Shah, K. \& Hansen, E. 1995 c Satellite and surface temperature data at odds? Clim. Change 30, 103-117.

Hansen, J., Ruedy, R., Sato, M. \& Reynolds, R. $1996 a$ Global surface air temperature in 1995: return to prePinatubo level. Geophys. Res. Lett. 23, 1665-1668.

Hansen, J. et al. $1996 b$ A Pinatubo climate modeling investigation. In NATO ASI Subseries I Global environment change (ed. G. Fiocco, D. Fua \& G. Visconti), pp. 233-272. Berlin: Springer-Verlag.

Hansen, J., Sato, M. \& Ruedy, R. 1997 a Radiative forcing and climate response. J. geophys. Res. (In the press.)

Hansen, J. et al. 1997 b Forcings and chaos in interannual to decadal climate change. J. geophys. Res. (Submitted).

Hayes, J. D., Imbrie, J. \& Shackleton, N. J. 1976 Variations in the Earth's orbit: pacemaker of the ice ages. Science, Wash. 194, 1121-1132.

Haywood, J. M. \& Shine, K. P. 1995 The effect of anthropogenic sulphate and soot aerosol on the clear sky planetary radiation budget. Geophys. Res. Lett. 22, 603-606.

Hobbs, P. V., Reid, J. S., Kotchenruther, R. A. \& Ferok, R. J. 1997 Optical parameters for smoke particles in Brazil and the direct radiative forcing of climate by smoke from biomass burning, Science, Wash. (Submitted).

Hoffert, M. I. \& Covey, C. 1992 Deriving global climate sensitivity from paleoclimate reconstructions. Nature, Lond. $360,573-576$.

Intergovernmental Panel on Climate Change 1996 Climate change 1995 (ed. J. T. Houghton, L. G. Meira Filho, B. A. Callander, N. Harris, A. Kattenberg \& K. Maskell). 572 pp. Cambridge University Press.

Karl, T. R., Jones, P. D., Knight, R. W. et al. 1993 A new perspective on recent global warming. Bull. Amer. Meteorol. Soc. 74, 1007-1023.

Karl, T. R., Knight, R. W., Kukla, G. \& Gavin, G. 1995 Evidence for the radiative effects of anthropogenic sulphate aerosols in the observed climate record. In Aerosol forcing of climate (ed. R. Charlson \& J. Heintzenberg), pp. 363-382. John Wiley \& Sons.

Lacis, A., Hansen, J. \& Sato, M. 1992 Climate forcing by stratospheric aerosols. Geophys. Res. Lett. 19, 1607-1610. 
Lean, J., Beer, J. \& Bradley, R. 1995 Reconstruction of solar irradiance since 1610: implications for climate change. Geophys. Res. Lett. 22, 3195-3198.

Liousse, C., Penner, J. E., Chuang, C., Walton, J.J. \& Cachier, H. 1996 A global three-dimensional model study of carbonaceous aerosols. J. Geophys. Res. 101, 19411-19432.

Lorius, C., Jouzel, J., Raynaud, D., Hansen, J. \& Le Treut, H. 1990 The ice-core record: climate sensitivity and future greenhouse warming. Nature, Lond. 347, 139-145.

Manabe, S. \& Stouffer, R. J. 1996 Low-frequency variability of surface air temperature in a 1000-year integration of a coupled atmosphere-ocean-land surface model. J. Climate 9, 376-393.

Mass, C. \& Schneider, S. H. 1978 Statistical evidence on the influence of sunspots and volcanic dust on long-term temperature trends. J. Atmos. Sci. 34, 1995-2004.

Matthews, E. 1983 Global vegetation and land-use: new high resolution data bases for climate studies. J. Clim. Appl. Meteorol. 22, 474- 487.

McCormick, M. P., Veiga, R. E. \& Chu, W. P. 1992 Stratospheric ozone profile and total ozone trends derived from the SAGE I and SAGE II data. Geophys. Res. Lett. 19, 269-272.

Minnis, P., Harrison, E. F., Stowe, L. L., Gibson, G. G., Denn, F. M., Doelling, D. R. \& Smith, F. L. 1993 Radiative climate forcing by the Mount Pinatubo eruption. Science, Wash. 259, 1411-1415.

Mishchenko, M. I. \& Travis, L. D. 1997 Satellite retrieval of aerosol properties over the ocean using polarization as well as intensity of reflected sunlight. J. Geophys. Res. (In the press.)

Mitchell, J. F. B., Johns, T. C., Gregory, J. M. \& Tett, S. F. B. 1995 Climate response to increasing levels of greenhouse gases and sulphate aerosols. Nature, Lond. 376, 501-504.

Mitchell, J. M. 1961 Recent secular changes of global temperature. Ann. N.Y. Acad Sci. 95, 235-250.

Ogren, J. A. 1995 A systematic approach to in situ observations of aerosol properties. In Aerosol forcing of climate (ed. R. Charlson \& J. Heintzenberg), pp. 215-226. John Wiley \& Sons.

Overpeck, J., Rind, D., Lacis, A. \& Healy, R. 1996 Tropospheric aerosols and abrupt climatic change during glacial periods. Nature, Lond. 447-449.

Parker, D. E., Wilson, H., Jones, P. D., Christy, J. R. \& Folland, C. K. 1996 The impact of Mount Pinatubo on worldwide temperatures. Internat. J. Climatol. 16, 487-497.

Penner, J. E., Dickinson, R. E. \& O’Neill, C. A. 1992 Effects of aerosol from biomass burning on the global radiation budget. Science, Wash. 256, 1432-1434.

Ramaswamy, V., Schwarzkopf, M. D. \& Randel, W.J. 1996 Fingerprint of ozone depletion in the spatial and temporal pattern of recent lower-stratospheric cooling. Nature, Lond. 382, 616-618.

Reichardt, J., Ansmann, A., Serwazi, M., Weitkamp, C. \& Michaelis, W. 1996 Unexpectedly low ozone concentration in midlatitude tropospheric ice clouds: a case study. Geophys. Res. Lett. 23, 1929-1932.
Rind, D. \& Peteet, D. 1985 Terrestrial conditions at the last glacial maximum and CLIMAP sea-surface temperature estimates: are they consistent? Quater. Res. 24, 1-22.

Santer, B. D. et al. 1996 A search for human influences on the thermal structure of the atmosphere. Nature, Lond. 382, 39-46.

Schrag, D. P., Hampt, G. H. \& Murray, D. W. 1996 Pore fluid constraints on the temperature and oxygen isotopic composition of the glacial ocean. Science, Wash. 272, 1930-1932.

Self, S. \& Rampino, M.R. 1988 The relationship between volcanic eruptions and climatic change: still a conundrum? Eos 69, 74-86.

Sokolik, I. N. \& Toon, O. B. 1996 Direct radiative forcing by anthropogenic airborne mineral aerosols. Nature, Lond. 381, 681-683.

Spencer, R. W. \& Christy, J. R. 1993 Precision lower stratospheric temperature monitoring with the MSU. J. Climate 6, 1194-1204.

Tegen, I., Lacis, A. A. \& Fung, I. 1996 The influence on climate forcing of mineral aerosols from disturbed soils. Nature, Lond. 380, 419-422.

\section{Discussion}

J. LeLieveld (Institute for Marine and Atmospheric Research, Utrecht, The Netherlands). TOMS/SAGE/sonde ozone measurements indicate ozone reductions in part of the atmosphere that is most sensitive to ozone forcing (free troposphere and lower stratosphere). Other information (e.g. in WMO 1995) suggests that ozone is increasing in the free troposphere. Could it be that the period before TOMS/ SAGE was characterized by ozone increases while after that (the past decade or so) stratospheric ozone depletion was dominant (also in the free troposphere)?

J. E. Hansen. I believe your latter suggestion is basically correct. Tropospheric ozone increased over the past century, and in some regions tropospheric ozone sources must be continuing to increase. But the ozone depletion in the lower stratosphere and tropopause region since the late 1970s should tend to decrease tropospheric ozone, especially in the upper troposphere. In our simulations for 1979-1995, we have a small ozone increase near the surface but a decrease in the upper troposphere, and thus we find a cooling in the tropopause region.

J. LeLieveld. Karl \& Kukla showed that the daytime maximum temperature has only marginally increased over continents, while the night-time minimum temperature has increased significantly. Can this be attributed to direct aerosol forcing, possibly in addition to changes in cloudiness?

J. E. Hansen. Aerosols themselves cannot be responsible, as we showed recently (Hansen, J. et al. 1995, Atmos. Res., 37, 175-209). Although aerosols reduce daytime heating and night-time cooling of the surface, the effect is much smaller than observed. We present evidence that the observed damping of the diurnal cycle implies an increase in continental cloud cover over the period 1950-1990. The increase may be an indirect effect of increasing aerosols. 